\title{
Chronic Cadmium Exposure Impairs Macrophage Mitochondrial Homeostasis and Promotes Macrophage Polarization Contributing to Atherosclerosis via Regulating RIPK3 Signaling
}

Jiexin Zhang

Southern Medical University

Weijing Feng

Southern Medical University

Peier Chen

Southern Medical University

Xiaodong Ning

Southern Medical University

Caiwen Ou

Southern Medical University

Minsheng Chen ( $\nabla$ minshengsmu@163.com)

Southern Medical University https://orcid.org/0000-0002-8271-268X

\section{Research}

Keywords: cadmium (Cd), atherosclerosis, RIPK3, macrophage polarization, mitochondrial homeostasis

Posted Date: April 14th, 2021

DOI: https://doi.org/10.21203/rs.3.rs-406704/v1

License: (c) (i) This work is licensed under a Creative Commons Attribution 4.0 International License.

Read Full License 


\section{Abstract}

Background: Chronic cadmium (Cd) exposure can contribute to the progression of cardiovascular disease (CVD), especially atherosclerosis (AS), but the underlying mechanism is unclear. Since mitochondrial homeostasis is emerging as a core player in the development of CVD, it might serve as a potential mechanism linking Cd exposure and AS. Here, we aimed to investigate the Cd-induced AS through macrophage polarization and tried to find out the mechanism of mitochondrial dysfunction caused by Cd exposure.

Methods and results: In vitro, flow cytometry showed that Cd exposure markedly promoted M1-type polarization of macrophages, manifesting as the increasing expression of NF-kB, NLRP3 and their downstream inflammatory factors, IL-1 $\beta$ and IL-6. Mitochondrial function test revealed that the decreasing mitochondrial membrane potential and increasing superoxide (mROS) and mitochondrial fission were involved in Cd-induced macrophage polarization. Transmission electron microscope observation and immunofluorescence both identified the decrease of mitophage after $\mathrm{Cd}$ exposure. And improving mitochondrial function above significantly restored the balance of macrophage polarization. In vivo, Cd exposure was positively correlated with blood $\mathrm{Cd}$ concentration, and oil red $\mathrm{O}$ staining showed higher blood Cd significantly increased the area of AS plaques. Besides, M1-type polarization of macrophages and mitochondrial dysfunction were observed in mouse aortic roots through immunofluorescence and western blot as the dosage of Cd increasing. And the administered NAC or Mdivi-1, which decreased mROS or mitochondrial fission, markedly attenuated AS plaques and macrophage M1-type polarization in Cd-treated group. Finally, the up-regulated expressions of RIPK3 and $\mathrm{p}-\mathrm{MLKL}$ were observed both in vitro and in vivo. And knocking out RIPK3 with decreasing expression of $\mathrm{p}$ MLKL followed did improve mitochondrial dysfunction caused by $\mathrm{Cd}$ which effectively reversed macrophage polarization.

Conclusion: Cd exposure activated RIPK3 pathway and impaired mitochondrial homeostasis, resulting in macrophage polarization to a pro-inflammatory phenotype and subsequent AS. These findings suggest that improving mitochondrial homeostasis may provide a potential therapeutic target for AS induced by chronic Cd exposure.

\section{Background}

With the rapid development of industry, Cd emerged as a common heavy metal pollutant in global environment. Because of the half-life of 10-35 years in humans and animals, $\mathrm{Cd}$ can cause serious damage to the body through biological amplification and accumulation [1]. A large body of crosssectional studies have shown that Cd exposure is associated with cardiovascular disease (CVD), such as atherosclerosis (AS) [2], coronary heart disease [3], peripheral arterial disease (PAD) [4], etc. Recent study found that blood $\mathrm{Cd}$ and urinary Cd levels were positively correlated with the occurrence of AS plaques, and the content of $\mathrm{Cd}$ in plaques was significantly higher than that in blood [5]. However, the exactly mechanism of Cd-induced AS is not clear. 
Macrophages are the largest number of immune cells in the cardiovascular system, with functional

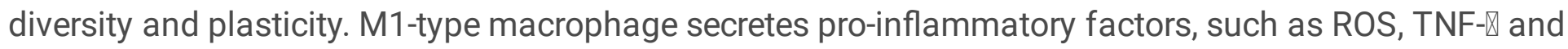
$\mathrm{IL}-1 \beta$, which is well documented to damage the AS plaque stability and cause stroke [6]. On the contrary, IL-10, FGF-1, IGF-1, TGF- $\beta$ and IL-12 released by M2-type macrophage contributes to the enhancement of AS plaque stability [7]. Additionally, mitochondrial membrane dynamics is tightly coupled to constant reshaping of the cellular mitochondrial network in a series of processes, involving organelle fusion and fission (division) as well as ultrastructural remodeling of the membrane [8]. And evidences accumulated has proved that the imbalance of mitochondrial homeostasis is the initial factors of macrophage dysfunction, which emerges as the impairment of protective autophagy [9] and promotes an inflammatory pathway [10]. Given all these, it is much meaningful to investigate whether Cd exposure affects the mitochondrial homeostasis of macrophages and further promotes M1-type polarization of macrophages in AS.

In addition, as a member of the receptor-interacting protein (RIP) family of serine/threonine protein kinases, receptor interacting serine/threonine kinase 3 (RIPK3) is closely related to inflammatory activation, which contributes to the expression of NF-KB transcription factor [11]. And as we knew, the inhibition of RIPK3 suppresses RNA virus-induced activation of the NLRP3 inflammasome [12]. On the aspect of membrane integrity, p-MLKL, phosphorylated by RIPK3, transfers from cytosol to cell plasma membrane and organelles' membranes, where it directly disrupts membrane integrity [13]. Additionally, RIPK1/RIPK3 complex directly phosphorylates Drp1 at serine 616 site (p-Drp1 ${ }^{(\text {Ser616) }}$ ) and triggers its translocation to mitochondria, finally increasing mitochondrial division [14]. Prior studies have suggested that RIPK3-knock-out can reduce plaque formation in the advanced stage of AS [15]. Therefore, whether RIPK3 was involved in Cd-induced macrophage mitochondrial membrane dynamic imbalance and M1type polarization in the progression of AS was a question to be studied in this research.

Thus, we hypothesized that high level blood Cd concentration could increase the expression of RIPK3-PMLKL in macrophages and make Drp1 transfer to mitochondria, causing macrophage mitochondrial dysfunction, triggering inflammation and M1-type polarization of macrophage, finally aggravating the progression of AS.

\section{Method}

\section{Cell culture}

RAW264.7 macrophages were cultured in Rosewell Park Memorial Institute (RPMI) 1640 medium supplemented with $20 \%$ fetal bovine serum (FBS), $100 \mathrm{U} / \mathrm{ml}$ penicillin and $100 \mathrm{mg} / \mathrm{ml}$ streptomycin at 37 ${ }^{\circ} \mathrm{C}$ in a humidified atmosphere with $5 \% \mathrm{CO}_{2}$ and maintained in a logarithmic growth phase for all experiments, then RAW264.7 macrophages were exposed to $\mathrm{CdCl}_{2}$ at different concentrations on the second day. To study the effect of mitochondrial function on the macrophages, we added $5 \mathrm{mM} \mathrm{N}$-Acetyl-

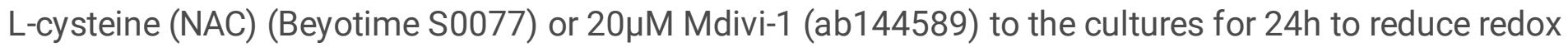


reactions or mitochondria division. What's more, bone marrow-derived macrophages (BMDMs) were isolated from RIPK3 ${ }^{-/} / \mathrm{ApoE}^{-/}$mice to research the effects of RIPK3 on Cd-exposed macrophages.

\section{Animal models of AS}

Animal experiments were carried out according to the National Institutes of Health Guidelines on the Use of Laboratory Animals and were approved by the Animal Ethics Committee of Southern Medical University. Seven-week-old male ApoE $E^{-/-}$mice were fed with high-fat diets or chow diets which were provided by Guangdong Medical Laboratory Animal Center for three months, exposed to Cd in different concentrations. To study the effect of Mitochondrial function on the formation of AS plaque, we administered NAC orally $(20 \mathrm{mM})$ or $50 \mathrm{mg} / \mathrm{kg}$ i.p.Mdivi- 1 to mice exposed to high-fat with $\mathrm{Cd}$ diet. And RIPK3-knockout mice on a C57BL/ 6 background were obtained from GemPharmatech, then bred with ApoE ${ }^{-/}$mice to establish RIPK3 ${ }^{-/ /} / \mathrm{ApoE}^{-/}$-mice.

\section{Tissue collection and analysis}

After fed with high-fat or chow diet for three months, mice were sacrificed with deep anesthesia for blood collection. Plasma was obtained from the blood samples of mice by centrifugation (Thermo Scientific ${ }^{\text {TM }}$ Medifuge $^{\mathrm{TM}}$ ) at $1000 \mathrm{xg}$ for 15 minutes at $4^{\circ} \mathrm{C}$ and then stored at $-80^{\circ} \mathrm{C}$. The mice were subsequently fixed by perfusion through the cardiac apex with phosphate buffer saline (PBS), and specimens of the aortic root were obtained without peripheral adipose tissue.

\section{CCK-8 (Cytotoxicity Assay)}

RAW264.7 cells in logarithmic growth phase were inoculated in a 96-well plate according to 10000 cells/well. The next day, the macrophages were observed growing to $70 \%$, then treated with different concentration gradients of $\mathrm{CdCl}_{2}$ for $24 \mathrm{~h}$. And the cell viability was detected by CCK-8 kit purchased from Dojindo (CKO4) in Plate Reader (Bio Tek Instruments Epoch ${ }^{\text {TM}}$ ).

\section{RNA extraction, reverse transcription, and real-time quantitative polymerase chain reaction (qRT-PCR)}

Total RNA was extracted from macrophages using Trizol reagent (Invitrogen 15596026). RNA was quantified and reverse-transcribed into complementary DNAs using PrimeScriptTM RT Master Mix kit (TaKaRa RR036A). Finally, the quantitative real-time PCR analysis (qRT-PCR) was performed using a TB Green Premix Ex TaqTMII (TaKaRa RR820A) kit on a Light Cycler96 PCR instrument (Roche). The Vazyme cycling conditions were: $95^{\circ} \mathrm{C}$ for $30 \mathrm{~s}$ followed by 39 cycles at $95^{\circ} \mathrm{C}$ for $10 \mathrm{~s}$ and $60^{\circ} \mathrm{C}$ for $30 \mathrm{~s}$. Then, a melting curve analysis was performed by increasing the temperature from $65^{\circ} \mathrm{C}$ to $95^{\circ} \mathrm{C}$ for $15 \mathrm{~min}$. GAPDH was used as a loading control. PCR primers used in this study were synthesized by TSINGKE (Beijing, China) and the sequences were: GAPDH (CATCACTGCCACCCAGAAGACTG (F), ATGCCAGTGAGCTTCCCGTTCAG (R)), IL-1ß(TGGACCTTCCAGGATGAGGACA (F), GTTCATCTCGGAGCCTGTAGTG (R)), IL-6 (TACCACTTCACAAGTCGGAGGC (F), CTGCAAGTGCATCATCGTTGTTC (R)), IL-10 (CGGGAAGACAATAACTGCACCC (F), 
CGGTTAGCAGTATGTTGTCCAGC (R)), TGF-ß(CGAAGCGGACTACTATGCTAAA (F), TCCCGAATGTCTGACGTATTG (R)), CD86 (ACGTATTGGAAGGAGATTACAGCT (F), TCTGTCAGCGTTACTATCCCGC (R)), CD206 (GTTCACCTGGAGTGATGGTTCTC (F), AGGACATGCCAGGGTCACCTTT (R)).

\section{Western blot}

Proteins were extracted from cells, tissues or mitochondria, while the mitochondria were isolated from RAW264.7 macrophages or aortic root using the cell mitochondria isolation kit (Beyotime C3601) or tissue mitochondria isolation kit (Beyotime C3606). After the proteins were measured by BCA (Beyotime Biotechnology, Beijing, CHINA) to the same concentration in each group, we boiled the proteins for 10 minutes, and separated it by $10 \%$ sodium dodecyl sulfatepolyacrylamide (SDS-PAGE) gel electrophoresis then transferred it onto a polyvinylidene difluoride (PVDF) membrane. After blocking the nonspecific binding sites with $5 \%$ non-fat milk in Tris buffered saline-Tween 20 (TBS-T), we incubated the membranes overnight with antibodies. The following primary antibodies were used: rabbit polyclonal antibody against GAPDH (ab9485), rabbit polyclonal antibody against NLRP3 (ab214185), rabbit monoclonal antibody against IL-1 beta[EPR16805-15] (ab234437), rabbit monoclonal antibody against TNF alpha [EPR19147] (ab183218), rabbit monoclonal antibody against IL-6 [EPR21710] (ab229381), rabbit monoclonal antibody against Opa1[EPR11057(B)] (ab157457), rabbit monoclonal antibody against MLKL (phospho S345) [EPR9515(2)] (ab196436) and rabbit monoclonal antibody against RIP3 (phospho S232) [EPR9516(N)-25] (ab195117) were from Abcam. Rabbit monoclonal antibody against Phospho-NFkappaB p65 (Ser536) (93H1)(Cell Signaling, \#3033S)『rabbit monoclonal antibody against Cleaved Caspase-1 (Asp296) (E2G2I) (Cell Signaling,\#89332S) هrabbit monoclonal antibody against COX IV (D6I4K) (Rodent Specific) (Cell Signaling, \#38563) were from Cell Signaling Technology. Rabbit polyclonal antibody against LC3I/II (WL01506) and rabbit polyclonal antibody against Drp1 (WL03028) were from Wanlei Biology. After having been incubated with the corresponding secondary antibodies (Boster BA1054) for 1.5 hours at room temperature, the immunoblot bands were detected by enhanced chemiluminescence (Engreen 29100). Prestained molecular-weight marker proteins (Thermo\#26616) were used to calculate the molecular weights of proteins. The membranes were exposed to autoradiography film following incubation with an enhanced chemiluminescence imaging system (Engreen 29100). The signal intensities were checked by Gel-Pro Analyzer 4.0 software (Media Cybernetics, Silver Spring, MD).

\section{Enzyme-linked immunosorbent assay (ELISA)}

The levels of inflammation markers in cell supernatant or mouse plasma, including interleukin $1 \beta$ (IL-1 $\beta$ ) and interleukin 6 (IL-6) were examined using ELISA kits (DAKEWE \#1210122 \#1210602).

\section{Flow Cytometry}

Cell surfaces were stained with phycoerythrin (PE)-conjugated anti-mouse F4/80 antibody (biolegend 123110), APC-conjugated anti-mouse CD206 (MMR) antibody (141708) and FITC-conjugated anti-mouse 
CD86 antibody (105006). The expression of F4/80 was determined by flow cytometry to identify macrophages. And the expression of CD86 was used to delineate M1 macrophages, while the cells stained by APC-conjugated anti-mouse CD206 (MMR) antibody could be identified as M2 macrophages. Cell suspensions were stained for 30 minutes on ice with specific antibodies and washed twice with $3 \mathrm{~mL}$ of PBS buffer supplemented with $0.5 \%$ bovine serum albumin (BSA). Finally, we analyzed it using flow cytometry (CytoFlex A00-1-1102).

\section{Immunofluorescence}

Cultured Raw264.7 macrophages or aortic root tissues were fixed with 4\% paraformaldehyde, incubated in $1 \%$ Triton for $10 \mathrm{~min}$ and then in $2 \%$ BSA for $1 \mathrm{~h}$, successively incubated with primary antibodies overnight at $4{ }^{\circ} \mathrm{C}$, followed by incubation with secondary antibodies. The following primary antibodies were used: F4/80 (BM8.1) rat mAb (\#71299S) from Cell Signaling Technology, CD86 Polyclonal rabbit antibody (13395-1-AP), CD206 Polyclonal rabbit antibody (18704-1-AP), Tom20 Polyclonal rabbit antibody (11802-1-AP) and LC3B-Specific Polyclonal rabbit antibody (18725-1-AP) from Proteintech. Then, the samples were stained with DAPI to visualize nucleus and observed by a laser confocal microscope (LEICA SP8). All the images and staining intensities were acquired and measured using analysis software (Image $\mathrm{J}$ ).

\section{Mitochondrial function detection}

The mitochondrial superoxide of Raw264.7 macrophages was detected by MitoSOX ${ }^{\mathrm{TM}}$ Red mitochondrial superoxide indicator (Invitrogen ${ }^{\text {TM }} \mathrm{M} 36008$ ), and the Mitochondrial membrane potential was determined via mitochondrial membrane potential assay kit with JC-1 (Beyotime C2006) according to the manufacture's instruction.

\section{Transmission electron microscope (TEM) observations}

Macrophages growing to the logarithmic phase were scraped and collected to be fixed with glutaraldehyde for 4 hours. Then sections $(60 \mathrm{~nm})$ were cut and stained with lead citrate and uranyl acetate at room temperature for $4 \mathrm{~h}$. Next, samples were embedded in resin at room temperature for another 2 hours. A Hitachi H-7500 Transmission Electron Microscope (Hitachi, Ltd., Tokyo Japan) was used to observe the samples.

\section{Oil Red 0 Staining}

After the serial $10 \mu \mathrm{m}$ sections were cut, the OCT compound-embedded tissues were stained with Oil Red O (Solarbio 08010) to evaluate the lipid content, and Re-dyed with hematoxylin (Beyotime), differentiated by hydrochloric alcohol (Beyotime), washed by double distilled water, finally observed under microscope (OLYMPUS).

\section{Quantification of blood Cd}


We added $500 \mu \mathrm{L} 1 \%$ HNO3 to $250 \mu \mathrm{L}$ whole blood of mice and added $0.01 \%$ TritonX-100 (Amresco 0694) to $5 \mathrm{~mL}$. Then we measured the concentration of blood Cd by ICP-Mass Spectrometer (PerkinElmer NexIONTM 350X).

\section{Statistical analyses}

Data were generated through at least three independent experiments and were presented as the means \pm SEM, then analyzed with the method of t-test between two groups or one-way ANOVA followed by a Bonferroni comparison test among three groups. Statistical analyses were carried out using Prism 8 (GraphPad). A two-tailed P value $<0.05$ was considered to be statistically significant.

\section{Results}

\section{Cd promoted macrophage polarization to a pro-inflammatory phenotype}

To evaluate the cytotoxicity caused by Cd, CCK8 measurement was performed after exposing RAW264.7 macrophages at logarithmic growth to $\mathrm{CdCl}_{2}$. Cell viability of RAW264.7 macrophages was more than $90 \%$ when exposed to $\mathrm{CdCl}_{2}$ with the concentration of $\leq 10 \mu \mathrm{mol} / \mathrm{L}$ (Fig. 1A). Subsequently, the macrophages were treated with $0.0,1.0,5.0$, and $10.0 \mu \mathrm{mol} / \mathrm{L} \mathrm{CdCl}_{2}$ for $\mathrm{qRT}$-PCR detection of related inflammatory factors, and the results showed that the lower concentration of $\mathrm{CdCl}_{2}(1 \mu \mathrm{mol} / \mathrm{L})$ had the most obvious pro-inflammatory effect (Fig. 1B). To further verify the effect of Cd on macrophage polarization in AS, $1 \mu \mathrm{mol} / \mathrm{L}$ Cd exposure was used for subsequent assays, including flow cytometry, western blot (WB) and ELISA after RAW264.7 foamed with ox-LDL $(50 \mu \mathrm{g} / \mathrm{mL})$ for $24 \mathrm{~h}$ in vitro. Exposed to $\mathrm{Cd}$, the surface-specific molecule of M1-type macrophages, CD86, was markedly up-regulated while the surface-specific molecule of M2-type macrophages, CD206, was significantly down-regulated (Fig. 1C and D). Then, the results of WB showed that the expressions of inflammasome NLRP3 and its downstream proteins, IL-1 and caspase1-p20, were increased, and the expressions of pNF-KB and its downstream proteins, TNF-a and IL-6, were also increased after Cd exposure (Fig. 1E-H). The proteins mentioned above were closely connected with M1-type polarization of macrophages [16]. Finally, the inflammatory factors, IL-1 $\beta$ and IL-6, in supernatant of macrophages were increased after Cd exposure measured by ELISA kit (Fig. 1I and J). Taken together, Cd promoted macrophage polarization toward the pro-inflammatory phenotype.

\section{Cd disrupted mitochondrial homeostasis and protective autophagy}

To investigate the effects of $\mathrm{Cd}$ exposure on the mitochondrial homeostasis and function of macrophages, we performed immunofluorescence staining on macrophages with the mitochondrial membrane protein Tom20 antibody. Among the groups we detected, the fragmentation of mitochondria was markedly increased in Cd-exposed group (Fig. 2A). Subsequently, the MitoSOX ${ }^{\mathrm{TM}}$ Red mitochondrial superoxide indicator was used to investigate that $\mathrm{Cd}$ exposure enhanced the oxidative stress responses in RAW264.7 (Fig. 2B and C). Moreover, the Cd-induced mitochondrial membrane potential reduction was presented in Fig. 2D and E. In terms of molecular mechanism, WB results revealed that Cd exposure led to 
the increased expression of mitochondrial division protein Drp1, decreased fusion protein Opa1, and decreased protective autophagy (the ratio of LC3II/I) in macrophages (Fig. 2F-H). In order to further verify the decreased protective autophagy level induced by decreased homeostasis, we performed immunofluorescence staining with autophagy-related protein LC3II, and the results showed that protective autophagosomes in macrophages were significantly decreased under $\mathrm{Cd}$ exposure (supplementary Fig. S1A and B). Finally, autophagosomes observed under TEM were decreased with Cd exposure (supplementary Fig. S1C). Overall, we found Cd-induced macrophage dysfunction was characterized by mitochondrial membrane dynamic imbalance.

\section{Improving mitochondrial homeostasis inhibited macrophage polarization toward the pro-inflammatory phenotype in vitro}

Based on the above experimental results, we conducted repair interventions for mitochondrial damage caused by $\mathrm{Cd}$ exposure: the antioxidant $\mathrm{N}$-acetylcysteine (NAC) was used to alleviate mitochondrial superoxide (mROS) production, and Mdivi-1, a mitochondrial fission inhibitor, was used to inhibit the expression of Drp1 in RAW264.7. The results showed that the above interventions effectively reduced the mitochondrial fragment of Cd-exposed macrophages (Fig. 3A), decreased the production of mROS (supplementary Fig. S2A and B), and recovered the mitochondrial membrane potential (supplementary Fig. S2E and F), indicating the restoration of mitochondrial homeostasis and function. Subsequently, WB identified that NAC and Mdivi-1 decreased the expression of Drp1, and increased the expression of Opa1 and the ratio of LC3II/I (Fig. 3B-D). Meanwhile, increased autophagosomes were presented by immunofluorescence with LC3II antibody after treatment of NAC and Mdivi-1(supplementary Fig. S2C and D). And it was measured by TEM that the above interventions significantly increased the content of autophagosomes in macrophages, while the production of mitophagy was more obvious in the Mdivi-1intervened group (Fig. 3E). Finally, the decreased expression of CD86, and the increased expression of CD206 were both demonstrated by flow cytometry with the recovery of mitochondrial homeostasis and function (Fig. 3F and G). Moreover, the expression of NLRP3, pNF-KB and their downstream proteins, IL$1 \beta$ and TNF-a, decreased in Cd-exposed group treated with NAC and Mdivi-1 (supplementary Fig. S3A and $B)$. These results indicated that improving mitochondrial homeostasis with NAC or Mdivi-1 effectively reversed Cd-induced macrophage polarization toward the pro-inflammatory phenotype.

\section{Improving mitochondrial homeostasis counteracted atherosclerosis in vivo}

In order to demonstrate the role of mitochondrial dysfunction and polarization of macrophages in the progression of $\mathrm{Cd}$-induced $\mathrm{AS}, \mathrm{ApoE}^{-/-}$mice exposed to $\mathrm{Cd}$ were treated with NAC or Mdivi-1 in vivo. Results showed that the blood Cd concentration was proportional to $\mathrm{Cd}$ exposure level (supplementary Fig. S4A). Oil red $O$ staining of the aortic root revealed that the plaque area of the high-fat feeding group with $200 \mathrm{mg} / \mathrm{L}$ Cd exposure was significantly increased compared with those without $\mathrm{Cd}$ exposure. No plaque formation was observed in the chow-diet with $200 \mathrm{mg} / \mathrm{L} \mathrm{Cd}$ exposure group. Meanwhile, NAC and Mdivi-1 effectively reduced the plaque area of the high-fat group with Cd exposure (Fig. 4A and B). Additionally, we performed immunofluorescence staining on the aortic root with the CD86 and CD206 
antibodies, finding that high-fat feeding groups with elevated blood Cd level had more obvious M1-type polarization than those without $\mathrm{Cd}$ exposure. And the ratio of $\mathrm{CD} 86^{+} / \mathrm{F} 4 / 80^{+}$was further increased as the concentration of Cd exposure increased from $100 \mathrm{mg} / \mathrm{L}$ to $200 \mathrm{mg} / \mathrm{L}$. In contrast, treatment with NAC or Mdivi-1 significantly decreased M1-type polarization (the ratio of $\mathrm{CD}^{2} 6^{+} / \mathrm{F} 4 / 80^{+}$) (Fig. $4 \mathrm{C}$ and D), while increasing M2-type polarization (supplementary Fig. S4B and C). Additionally, mice treated with NAC or Mdivi-1 showed a significant increase in the expression of Opa1 and the ratio of LC3II/I but a remarked decrease in the expression of Drp1, NLRP3 and pNF-KB (Fig. 4E-G; supplementary Fig. S5A-G). Finally, plasma ELISA results showed the decreased level of M1-type inflammatory markers, il-1 and il-6, after NAC or Mdivi-1 treatment (supplementary Fig. S5H-I). Taken together, improving mitochondrial homeostasis inhibited Cd-induced macrophage polarization to the pro-inflammatory phenotype and thus counteracted AS in vivo.

\section{The level of RIPK3 was increased in atherosclerosis while RIPK3 knockout enhanced mitochondrial homeostasis}

Next, we wanted to further explore the deep mechanism of Cd-induced mitochondrial homeostasis injury. Firstly, we found that the expression of RIPK3 and its downstream protein, p-MLKL, were significantly increased under $\mathrm{Cd}$ exposure both in vivo and in vitro (Fig. 5A-C). Meanwhile, the expression of RIPK3 in mitochondria was decreased after the mitochondrial dysfunction was restored by NAC or Mdivi-1 (supplementary Fig. S6A-F), which could be explained that the translocation of necrosomes (RIPK1RIPK3-MLKL) from the cytosol to intracellular membranes was inhibited [17]. Then we extracted BMDMs from RIPK3 ${ }^{-/-} / \mathrm{ApoE}^{-/-}$mice, added ox-LDL to induce foam cell, and found the expression of $\mathrm{p}-\mathrm{MLKL}$ was decreased with the knockout of RIPK3 (supplementary Fig. S6G). Compared with ApoE ${ }^{-/-}$mice, BMDMs from RIPK3 ${ }^{-/-} / \mathrm{ApoE}^{-/-}$mice showed reduced mitochondrial fragmentation, increased mitochondrial membrane potential, and decreased mROS, indicating that mitochondrial membrane homeostasis and function were restored after Cd exposure (Fig. 5D-H). Moreover, WB results showed that knocking out RIPK3 decreased the expression of Drp1, increased the expression of Opa1, and the ratio of LC3II/I both in BMDMs and in aortic root (Fig. 5I-K) (supplementary Fig. S6J-L). And the immunofluorescence of LC3II showed that the number of autophagosomes was indeed increased in $\mathrm{RIPK}^{-/-} / \mathrm{ApoE}^{-/-}$mice (supplementary Fig. S6H and I). These results indicated that RIPK3-p-MLKL pathway was activated under Cd exposure, and RIPK3 knockout significantly enhanced mitochondrial homeostasis.

\section{Deletion of RIPK3 inhibited polarity shift toward inflammatory macrophages and atherosclerosis}

To understand the in vivo role of RIPK3 in the development of Cd-enhanced AS through mitochondrial homeostasis and macrophages inflammation, we employed the $\mathrm{ApoE}^{-/-}$AS mice with a deletion of RIPK3 (RIPK3 ${ }^{-/-} / \mathrm{ApoE}^{-/-}$). To accelerate AS, mice were fed a high-fat diet and exposed to Cd. Oil red $\mathrm{O}$ staining of the aortic root showed that the area of AS plaques was significant decreased in RIPK3 ${ }^{-/}$ -/ApoE ${ }^{-/-}$mice compared with $\mathrm{ApoE}^{-/-}$mice (Fig. 6A and B). Immunofluorescence staining of the aortic roots showed that the level of M1-type polarization $\left(\mathrm{CD} 86^{+} / \mathrm{F} 4 / 80^{+}\right)$decreased while the level of M2-type 
polarization $\left(\mathrm{CD} 206^{+} / \mathrm{F} 4 / 80^{+}\right)$increased in $\mathrm{RIPK}^{-/-} / \mathrm{ApoE}^{-/-}$mice compared to $\mathrm{ApoE}^{-/-}$mice (Fig. 6C-E). Additionally, deletion of RIPK3 inhibited polarity shift toward M1-type BMDMs shown by flow cytometry (Fig. 6F and G). The expressions of NLRP3 and pNF-KB were inhibited both in aortic root and in BMDMs of RIPK3 ${ }^{-/-} / \mathrm{ApoE}^{-/-}$mice (Fig. $6 \mathrm{H}$ and I; supplementary Fig. S7A and B). Plasma ELISA was conducted to investigate that the expressions of inflammatory cytokines, IL-1 $\beta$ and IL-6, secreted by M1-type macrophages were decreased in $\mathrm{RIPK}^{-/-} / \mathrm{ApoE}^{-/-}$mice, too (supplementary Fig. S7C). These results indicated that the deletion of RIPK3 inhibited Cd-induced inflammatory responses and subsequent AS.

\section{Discussion}

In this study, we focused on the non-essential elements of the human body, $\mathrm{Cd}$, which was widely existing in the nature. Our findings showed that $\mathrm{Cd}$ disrupted macrophage mitochondrial homeostasis through RIPK3 signaling pathway and caused M1-type polarization of macrophage, finally aggravating the progression of AS (Fig. 7). To the best of our knowledge, we demonstrated the first time that the mechanism of Cd-induced AS is the imbalance of macrophage mitochondrial homeostasis via RIPK3 pathway.

Previous studies on Cd-induced inflammation of macrophages reported that oral exposure to Cd (3 hours) triggered an acute inflammatory response in the intestines of mice, initiated by the over-expression of tissue macrophage inflammatory protein- 2 mRNA which was produced by both intestinal epithelial cells and macrophages. But this study showed no significant changes of cytokines, IL-1 $\beta$ and TNF-a [18]. In contrast, Marina Ninkov et al studied the effects of oral Cd exposure on intestinal immunity, suggesting that $\mathrm{Cd}$ consumption resulted in the changes of intestinal flora as reduction of Lactobacillus strain, and intestinal inflammation as increasing pro-inflammation cytokines (TNF, IL-1 $\beta$, IFN-y, IL-17) [19]. Furthermore, Kielldahl et al found that blood $\mathrm{Cd}$ was associated with pro-inflammatory macrophage density in the sections of carotid plaques with most frequent rupture which was previously shown to contain most $\mathrm{Cd}$ [20]. The studies mentioned above indicated that $\mathrm{Cd}$ can induce inflammatory response in macrophages which was the same as the result of this article. However, the pro-inflammatory properties of $\mathrm{Cd}$ is still controversial. Till now, the only one article that mentioned the effect of $\mathrm{Cd}$ on macrophage polarization revealed that in chronic obstructive pulmonary disease COPD patients, Cd exposure inhibited the NF-KB pathway, dose-dependently inhibited LPS-induced immunoreaction by macrophages, and inhibited M1-type macrophage behavior with less effect on M2-type polarization. This article became a new evidence that $\mathrm{Cd}$ could lead to immune dysfunction, further increasing the susceptibility to COPD infection [21]. Furthermore, another study came up to be the first report investigating the effects of $\mathrm{Cd}$ on the inflammatory responses and oxidative stress together in vitro system, and the result showed that murine macrophage exposed to Cd significantly decreased the inflammatory responses but increased the oxidative stress, a little different from studies explained above [22]. The difference may be due to $\mathrm{Cd}$ concentration and treatment time that high-level $\mathrm{Cd}$ exposure for long time can impair cell viability and function so that the high concentration of $\mathrm{Cd}$ in smokers' lungs impairs the normal pro-inflammatory function of macrophages and worsens the infection of COPD. 
However, the concentration of blood Cd is not high, as a result, the function of macrophages in cardiovascular system hasn't been impaired.

As to the mechanism of Cd-induced inflammation, an existing study on Cd-induced liver injury showed that liver injury was mediated by inflammatory mediators activated by reactive oxygen species [23]. Glycine, an antioxidant, could reduce the secretion of inflammatory cytokines IL-6, TNF- $\alpha$ and IL-1 by macrophage U937 cells [24]. In addition, another study has shown that the enhanced REDOX reaction in peritoneal macrophages of mice exposed to chronic low levels of $\mathrm{Cd}$ induced lipid peroxidation, and increased the expressions of cyclooxygenase- 2 and inducible nitric oxide synthase, finally causing inflammatory response [25]. Therefore, we can conclude that Cd-induced oxidative stress is related to inflammatory response, and the former is the upstream pathway of the latter which is the same as the result of our research. Furthermore, the main sources of intracellular ROS are membrane-derived and mitochondrion-derived. The former is mainly dependent on NAPDH oxidase, while the latter is associated with mitochondrial membrane potential damage. As to the mitochondrion-derived ROS, studies have shown that mitochondria in THP-1 macrophages could be targets of $\mathrm{Cd}$ toxicity: $\mathrm{Cd}$ binded to thiol proteins on the mitochondrial membrane, and then changed the mitochondrial membrane permeability to inhibit the mitochondrial respiratory chain response, or damaged the function of electron transfer chain complex III, and finally induced the production of ROS [26]. However, the mitochondrial damage and subsequent macrophage polarization involved in the process of Cd-induced AS remain unclear. In this study, we filled this gap in knowledge by reporting that chronic Cd exposure impaired macrophage mitochondrial homeostasis and promoted macrophage polarization contributing to AS through RIPK3 pathway.

The molecular mechanism of inflammatory reaction includes the discovery by Ja Shil Hyun et al in 2007 that $20-60 \mu \mathrm{M}$ concentration of $\mathrm{CdCl}_{2}$ significantly induced the increase of IL-8 secretion through NF-KB activation in human intestinal epithelial cell Caco-2 [27]. Moreover, vitro studies showed that Cd activated NF-KB pathway through increasing oxidative stress level [28]. In addition, Wirth's team has successively found that the activation of heat shock response induced by $\mathrm{Cd}$ effectively protect the lung function of mice. Compared with HSF-/- mice, NF-KB pathway was suppressed in wild-type mice, leading to lower infiltrating macrophages and neutrophils $[29,30]$. These studies showed that the activation of NF-KB was one of the molecular mechanisms by which Cd promoted inflammation, the same as our results. Besides, Kunpeng Wu's team found that the M1-type polarization of macrophages required NLRP3 inflammasome activation [16]. But no researches related to Cd-induced macrophage polarization have paid attention to NLRP3 inflammasome except this study.

In addition, as described above, RIPK3 was associated with mitochondrial function, and the expressions of NF-KB and NLRP3, but there has been no study revealing that Cd could impair macrophage mitochondrial homeostasis and promote macrophage polarization contributing to atherosclerosis via regulating RIPK3 signaling. So trying to verify the polarization effect of macrophages induced by Cd in AS, exploring the mechanism from the perspective of mitochondrial homeostasis and dysfunction, finally knocking out RIPK3 gene from the upstream of mitochondria, made this study more innovative. 
In addition to AS, Cd exposure can also worsen other CVD progression. It has been reported that Cd induced hypertension by decreased endothelial nitric oxide synthase protein level [31]. Additionally, macrophages create a beneficial microenvironment for the survival of myocardial cells, which is crucial for the survival and regeneration of myocardial cells after MI [32]. And local inflammatory response and changes in immune microenvironment are considered to be one of the main reasons for the difficulty in myocardial repair and regeneration after myocardial tissue injury [33]. Therefore, the influence of $\mathrm{Cd}$ exposure on the immune microenvironment in the heart region may be the key interference factor in the myocardial repair after MI in Cd-exposed area. At the same time, many known studies have reported that bone and kidney were two of the important target organs of multi-organ damage caused by Cd poisoning, among which osteoporosis is the main manifestation of bone damage caused by $\mathrm{Cd}$ [34]. Moreover, existing studies have shown that with the growth of age, the lost human calcium from bones would deposit on the cardiovascular system and then induce vascular calcification [35]. Although there is a strong relationship between osteoporosis and vascular calcification, there is a lack of research on the mechanism of vascular calcification caused by $\mathrm{Cd}$ exposure.

Finally, in addition to the environmental pollutant, $\mathrm{Cd}$, which was the focus of this paper, other heavy metal pollution, such as lead, mercury, and arsenic, may also have an impact on the cardiovascular system [36]. Will they have a synergistic effect with $\mathrm{Cd}$ ? Is there a natural substance (e.g., selenium) that can counteract the damage caused by Cd contamination? Further study need to be investigated.

\section{Conclusion}

Our findings demonstrated that mitochondrial homeostasis played an important role in Cd-induced inflammatory response in macrophages. We further revealed that RIPK3 regulated Cd-induced mitochondrial dysfunction via Drp1 protein, leading to increasing expressions of NF-KB and NLRP3 proteins. Finally, Cd exposure induced M1-type polarization of macrophages in cardiovascular system which was conducive to AS. These results revealed a novel mechanism for Cd-induced inflammation and offered new insights into the pathophysiology of AS caused by heavy metal pollution.

\section{Abbreviations}

AS: Atherosclerosis; BSA: Bovine serum albumin; CCK-8: Cytotoxicity assay; Cd: Cadmium; CVD: Cardiovascular disease; ELISA: Enzyme-linked immunosorbent assay; FBS: Fetal bovine serum; NAC: NAcetyl-L-cysteine; PAD: Peripheral arterial disease; PBS: Phosphate buffer saline; qRT-PCR: Real-time quantitative polymerase chain reaction; RIPK3: Receptor interacting serine/threonine kinase 3; TEM: Transmission electron microscope; WB: Western blot

\section{Declarations}

\section{Ethics approval and consent to participate}


Animal experiments were carried out according to the National Institutes of Health Guidelines on the Use of Laboratory Animals and were approved by the Animal Ethics Committee of Southern Medical University.

\section{Consent for publication}

Not applicable.

\section{Availability of data and materials}

All data and materials are included in the main body of the manuscript or in the additional files. The datasets generated and/or analyzed during the current study are available from the corresponding author on reasonable request.

\section{Competing interests}

The authors declare that they have no competing interests.

\section{Funding}

This work was supported by the National Natural Science Foundation of China (Nos. 31771099, 81871504, and 31771060ه81971765】

\section{Authors' contributions}

JZ: Conceptualization, Methodology, Validation, Investigation, Analysis of data, Writing (original draft), Visualization. WF: Methodology, Validation, Writing (review and editing), interpretation of data. PC: Methodology, Validation. XN: Methodology. CO: Methodology, Validation, Supervision, Writing (review and editing), Funding acquisition. MC: Methodology, Conceptualization, Supervision, Writing (review and editing), Funding acquisition. All authors read and approved the final manuscript.

\section{Acknowledgments}

Not applicable.

\section{References}

1. Satarug, S., et al., Cadmium, environmental exposure, and health outcomes. Environ Health Perspect, 2010. 118(2): p. 182-90.

2. Borné, Y., et al., Cadmium, Carotid Atherosclerosis, and Incidence of Ischemic Stroke. J Am Heart Assoc, 2017. 6(12).

3. Chowdhury, R., et al., Environmental toxic metal contaminants and risk of cardiovascular disease: systematic review and meta-analysis. Bmj, 2018. 362: p. k3310. 
4. Tellez-Plaza, M., et al., Cadmium exposure and incident peripheral arterial disease. Circ Cardiovasc Qual Outcomes, 2013. 6(6): p. 626-33.

5. Fagerberg, B., et al., Cadmium exposure and atherosclerotic carotid plaques-results from the Malmö diet and Cancer study. Environ Res, 2015. 136: p. 67-74.

6. Chistiakov, D.A., et al., Immune-Inflammatory Responses in Atherosclerosis: The Role of Myeloid Cells. J Clin Med, 2019. 8(11).

7. Jinnouchi, H., et al., Diversity of macrophage phenotypes and responses in atherosclerosis. Cell Mol Life Sci, 2020. 77(10): p. 1919-1932.

8. Giacomello, M., et al., The cell biology of mitochondrial membrane dynamics. Nature Reviews Molecular Cell Biology, 2020. 21(4): p. 204-224.

9. Zhong, J., et al., Therapeutic contribution of melatonin to the treatment of septic cardiomyopathy: A novel mechanism linking Ripk3-modified mitochondrial performance and endoplasmic reticulum function. Redox Biol, 2019. 26: p. 101287.

10. Bock, F.J. and S.W.G. Tait, Mitochondria as multifaceted regulators of cell death. Nature Reviews Molecular Cell Biology, 2020. 21(2): p. 85-100.

11. Yang, B. and L.A. Maddison, RIPK3-mediated inflammation is a conserved $\beta$ cell response to ER stress. 2020. 6(51).

12. Wang, X., et al., RNA viruses promote activation of the NLRP3 inflammasome through a RIP1-RIP3DRP1 signaling pathway. Nat Immunol, 2014. 15(12): p. 1126-33.

13. Pasparakis, M. and P. Vandenabeele, Necroptosis and its role in inflammation. Nature, 2015. 517(7534): p. 311-20.

14. Zhang, S., et al., Drp1 and RB interaction to mediate mitochondria-dependent necroptosis induced by cadmium in hepatocytes. Cell Death Dis, 2019. 10(7): p. 523.

15. Lin, J., et al., A role of RIP3-mediated macrophage necrosis in atherosclerosis development. Cell Rep, 2013. 3(1): p. 200-10.

16. Wu, K., et al., The gut microbial metabolite trimethylamine N-oxide aggravates GVHD by inducing M1 macrophage polarization in mice. Blood, 2020. 136(4): p. 501-515.

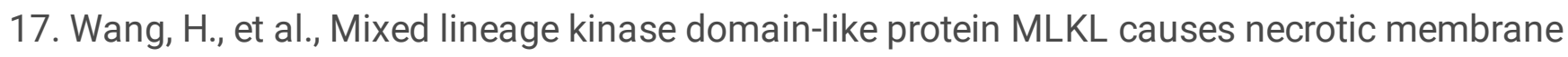
disruption upon phosphorylation by RIP3. Mol Cell, 2014. 54(1): p. 133-146.

18. Zhao, Z., et al., Oral exposure to cadmium chloride triggers an acute inflammatory response in the intestines of mice, initiated by the over-expression of tissue macrophage inflammatory protein-2 mRNA. Toxicol Lett, 2006. 164(2): p. 144-54.

19. Ninkov, M., et al., Toxicity of oral cadmium intake: Impact on gut immunity. Toxicol Lett, 2015. 237(2): p. 89-99.

20. Fagerberg, B., et al., Cadmium exposure as measured in blood in relation to macrophage density in symptomatic atherosclerotic plaques from human carotid artery. Atherosclerosis, 2016. 249: p. 209214. 
21. Cox, J.N., et al., Cadmium attenuates the macrophage response to LPS through inhibition of the NFKB pathway. Am J Physiol Lung Cell Mol Physiol, 2016. 311(4): p. L754-I765.

22. Jin, Y., et al., Cadmium exposure to murine macrophages decreases their inflammatory responses and increases their oxidative stress. Chemosphere, 2016. 144: p. 168-75.

23. Dong, W., et al., Toxic metals stimulate inflammatory cytokines in hepatocytes through oxidative stress mechanisms. Toxicol Appl Pharmacol, 1998. 151(2): p. 359-66.

24. Okoko, T. and E.P. Awhin, Glycine reduces cadmium-induced alterations in the viability and activation of macrophage U937 cells. Food Chem Toxicol, 2010. 48(2): p. 536-8.

25. Ramirez, D.C. and M.S. Gimenez, Induction of redox changes, inducible nitric oxide synthase and cyclooxygenase- 2 by chronic cadmium exposure in mouse peritoneal macrophages. Toxicology Letters, 2003. 145(2): p. 121-132.

26. Olszowski, T., et al., The Effects of Cadmium at Low Environmental Concentrations on THP-1 Macrophage Apoptosis. Int J Mol Sci, 2015. 16(9): p. 21410-27.

27. Hyun, J.S., H. Satsu, and M. Shimizu, Cadmium induces interleukin-8 production via NF-kappaB activation in the human intestinal epithelial cell, Caco-2. Cytokine, 2007. 37(1): p. 26-34.

28. Hart, B.A., et al., Characterization of cadmium-induced apoptosis in rat lung epithelial cells: evidence for the participation of oxidant stress. Toxicology, 1999. 133(1): p. 43-58.

29. Wirth, D., et al., Use of Hsf1(-/-) mice reveals an essential role for HSF1 to protect lung against cadmium-induced injury. Toxicol Appl Pharmacol, 2003. 192(1): p. 12-20.

30. Wirth, D., et al., Evidence for a role of heat shock factor 1 in inhibition of NF-kappaB pathway during heat shock response-mediated lung protection. Am J Physiol Lung Cell Mol Physiol, 2004. 287(5): p. L953-61.

31. Yoopan, N., et al., Attenuation of eNOS expression in cadmium-induced hypertensive rats. Toxicol Lett, 2008. 176(2): p. 157-61.

32. Gentek, R. and G. Hoeffel, The Innate Immune Response in Myocardial Infarction, Repair, and Regeneration. Adv Exp Med Biol, 2017. 1003: p. 251-272.

33. Notari, M., et al., The local microenvironment limits the regenerative potential of the mouse neonatal heart. Science advances, 2018. 4(5): p. eaao5553.

34. Chen, $X$., et al., Cadmium is more toxic on volume bone mineral density than tissue bone mineral density. Biol Trace Elem Res, 2011. 144(1-3): p. 380-7.

35. Zeng, Y., et al., Mechanical microenvironment regulation of age-related diseases involving degeneration of human skeletal and cardiovascular systems. Prog Biophys Mol Biol, 2019. 148: p. 54-59.

36. Chowdhury, R., et al., Environmental toxic metal contaminants and risk of cardiovascular disease: systematic review and meta-analysis. BMJ (Clinical research ed.), 2018. 362: p. k3310.

\section{Figures}



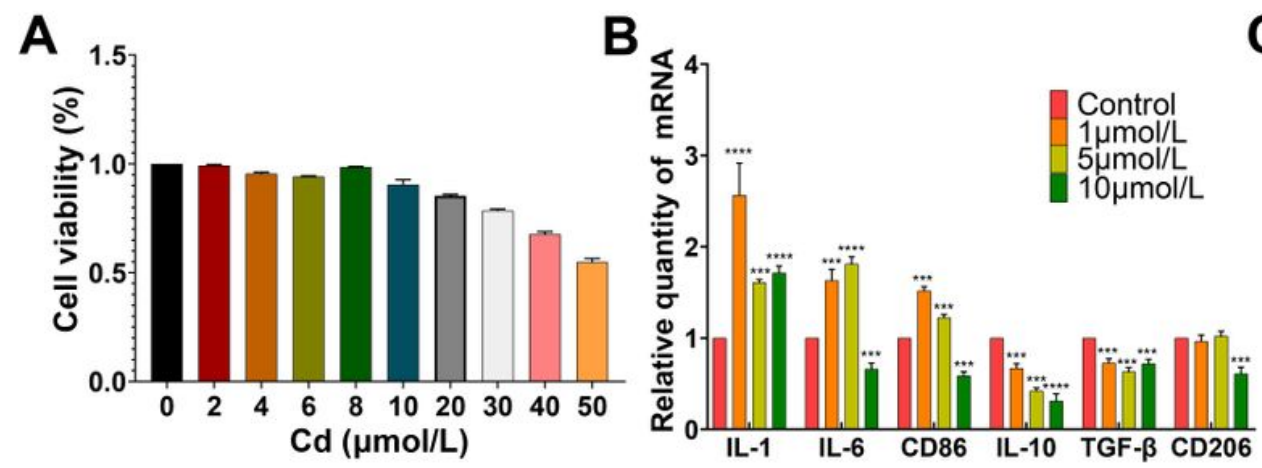

C
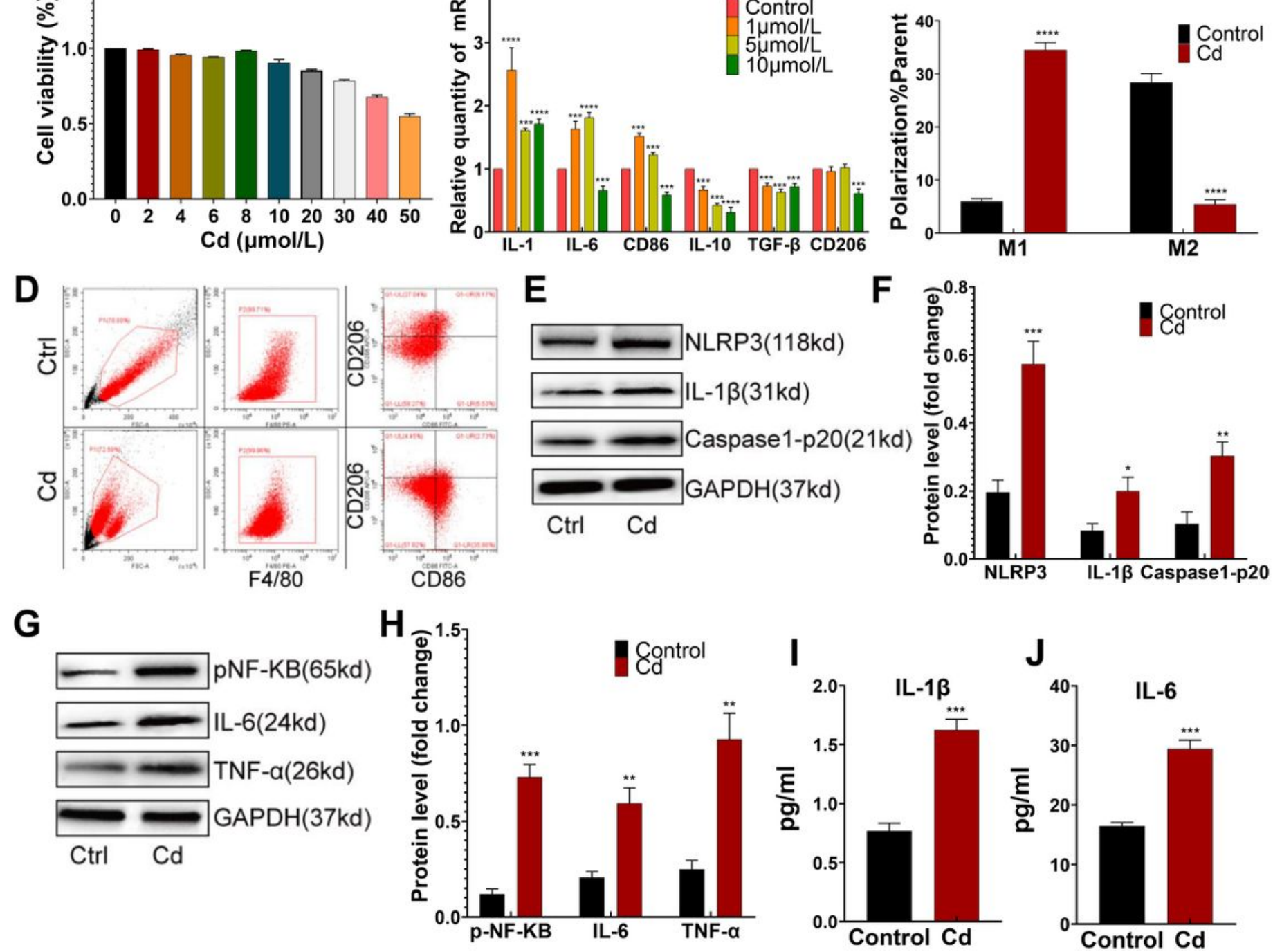

Figure 1

M1-type polarization of macrophage caused by $\mathrm{Cd}$. (A) The survival rate of macrophages exposed to different concentrations of Cd detected by CCK-8 kit. (B) The expression of mRNA related to Cd-induced inflammation measured by q-PCR. (C, D) The Cd-induced polarization of macrophages showed by flow cytometry. (E, F) The protein expression levels of NLRP3, IL-1 $\beta$ and Caspase1-p20 in RAW264.7 cells after treatment with Cd. $(\mathrm{G}, \mathrm{H})$ The protein expression levels of pNF-KB, IL-6 and TNF- $\alpha$ in RAW264.7 cells after treatment with $\mathrm{Cd}$. $(\mathrm{I}, \mathrm{J})$ The levels of IL-1 $\beta$ and IL- 6 in the supernatant of macrophages. Data are shown as mean \pm SD. ${ }^{\star} p<0.05,{ }^{\star *} p<0.01,{ }^{* * *} p<0.001,{ }^{\star \star * \star} p<0.0001$. 

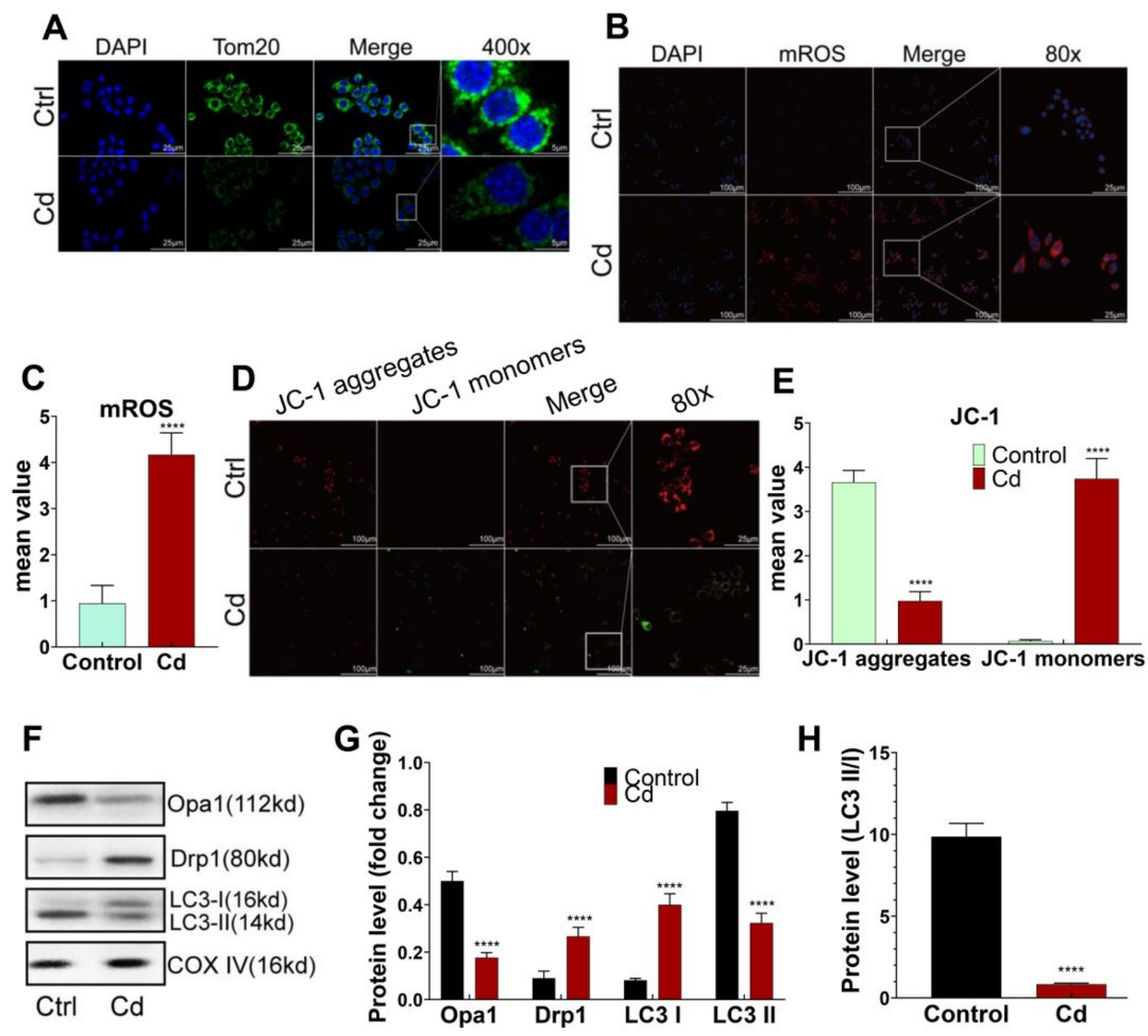

\section{Figure 2}

The changes of mitochondrial homeostasis and protective autophagy after $\mathrm{Cd}$ exposure. (A) Immunofluorescence images of mitochondrial membrane protein Tom20 in RAW264.7 cells after treatment with Cd. (B, C) Immunofluorescence images and fluorescence intensity of mitochondrial superoxide (mROS) in RAW264.7 cells after treatment with Cd. (D, E) Mitochondrial membrane potential showed by fluorescent probe (JC-1). (F) The protein expression levels of Opa1, Drp1, LC3I and LC3II in mitochondria of RAW264.7 cells after treatment with Cd. COX-IV is the internal reference of the mitochondrial protein. $(G)$ The gray value of the proteins mentioned above. $(H)$ The ratio of LC3II and LC3I indicating the autophagy level. Data are shown as mean \pm SD. ${ }^{\star} p<0.05,{ }^{\star *} p<0.01,{ }^{\star \star \star} p<0.001$, ${ }^{\star \star \star \star} p<$ 0.0001 . 


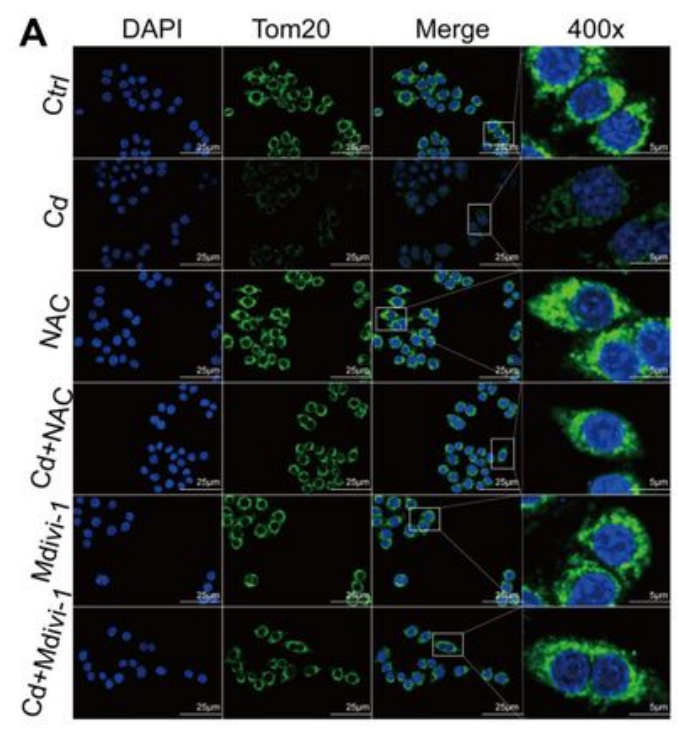

E
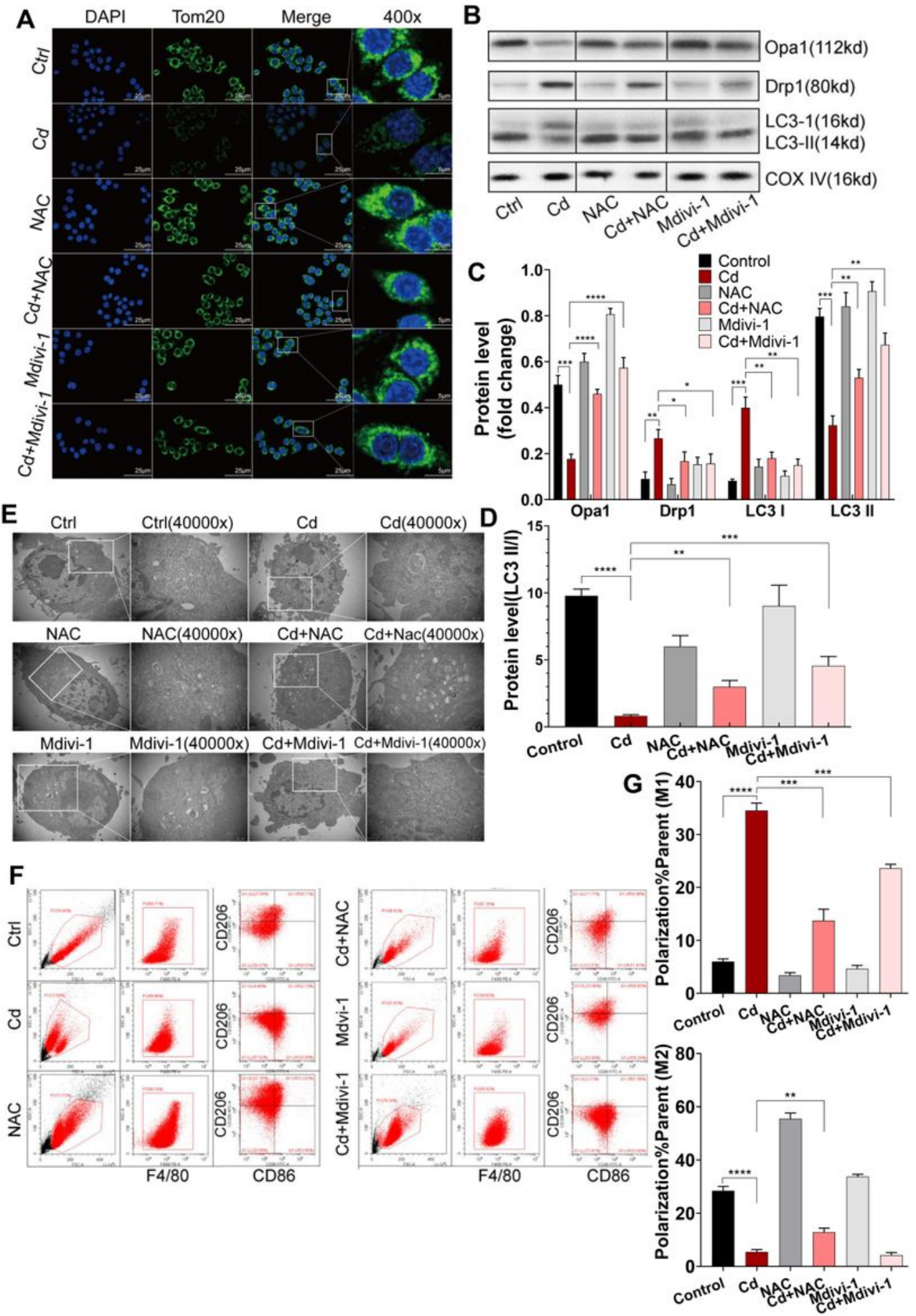

\section{Figure 3}

Improving mitochondrial homeostasis in the process of macrophage M1-type polarization in vitro. (A) Immunofluorescence images of mitochondrial membrance protein Tom20 in RAW264.7 cells. (B, C) The protein expression levels of Opa1, Drp1, LC3I and LC3II in mitochondria of RAW264.7 cells. (D) The ratio of LC3II and LC3I indicating the autophagy level. (E) Representative TEM images of autophagosome in RAW264.7 cells after treatment with Cd, NAC and Mdivi-1. (F, G) The polarization of macrophages were 
measured by flow cytometry. Data are shown as mean \pm SD. ${ }^{\star} p<0.05,{ }^{* \star} p<0.01,{ }^{\star \star \star} p<0.001,{ }^{* \star \star \star} p<$ 0.0001 .
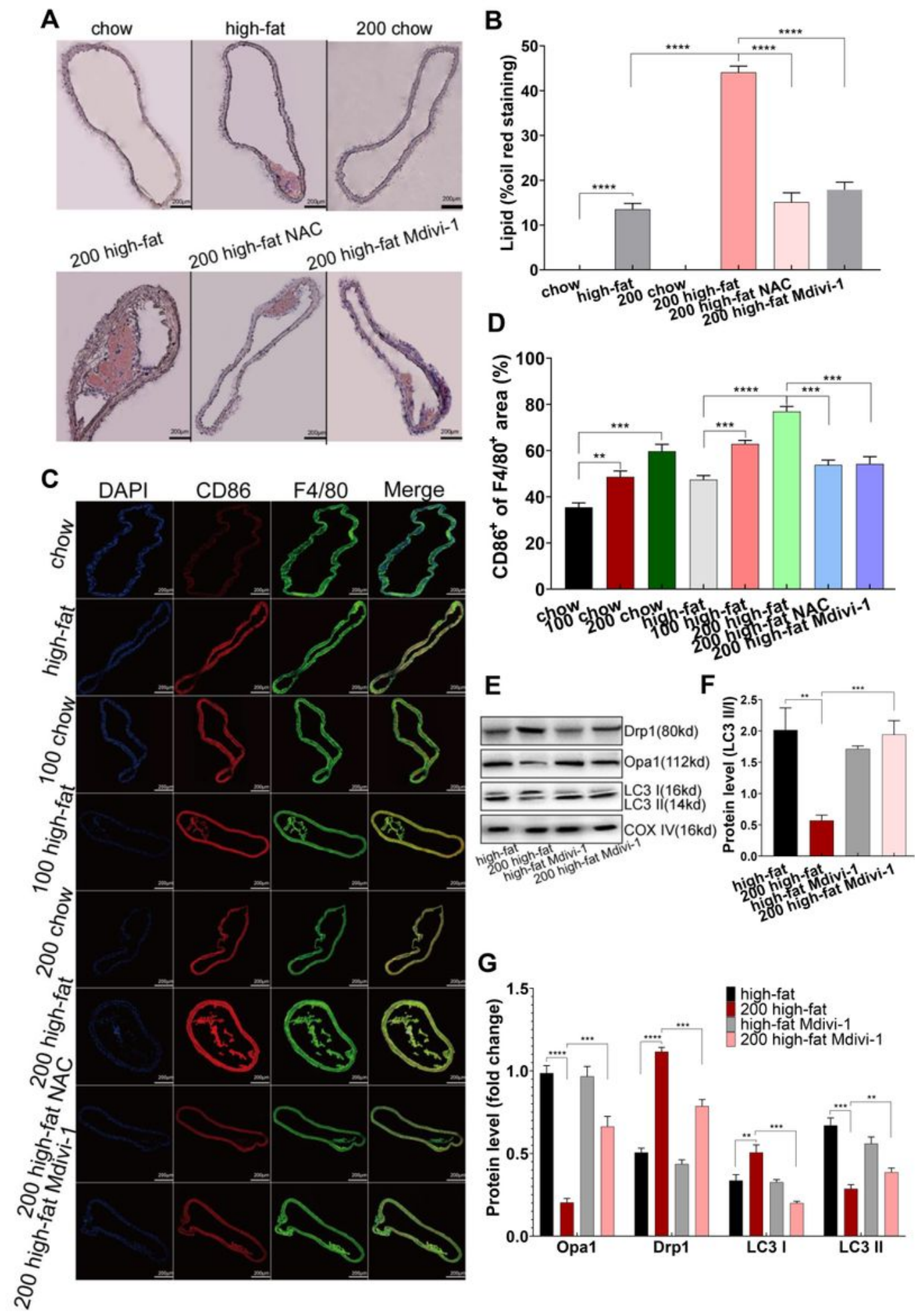

Figure 4

Mitochondrial homeostasis mediated inflammation of Cd-induced AS in vivo. (A) Representative photomicrographs of the aortic arch segments from $\mathrm{Cd}$ - and high-fat-treated ApoE-/-mice stained with Oil Red O. (B) Quantification of the atherosclerotic lipid content. (C) Aortic arch of Cd-, high-fat-, NAC- and 
Mdivi-1-treated ApoE-/- mice were probed with specific antibodies against the macrophage marker F4/80 and co-probed with antibodies against the markers of M1 (CD86). (D) Quantification of CD86+ of F4/80+ area in aortic arch. (E-G) The protein expression levels of Opa1, Drp1, LC3I and LC3II in mitochondria of mice aortic arch after treatment with $\mathrm{Cd}$, high-fat, and Mdivi-1. ( $\mathrm{n}=6-8$ per group). Data are shown as mean \pm SD. ${ }^{*} p<0.05,{ }^{* *} p<0.01, * * * p<0.001, * * * p<0.0001$

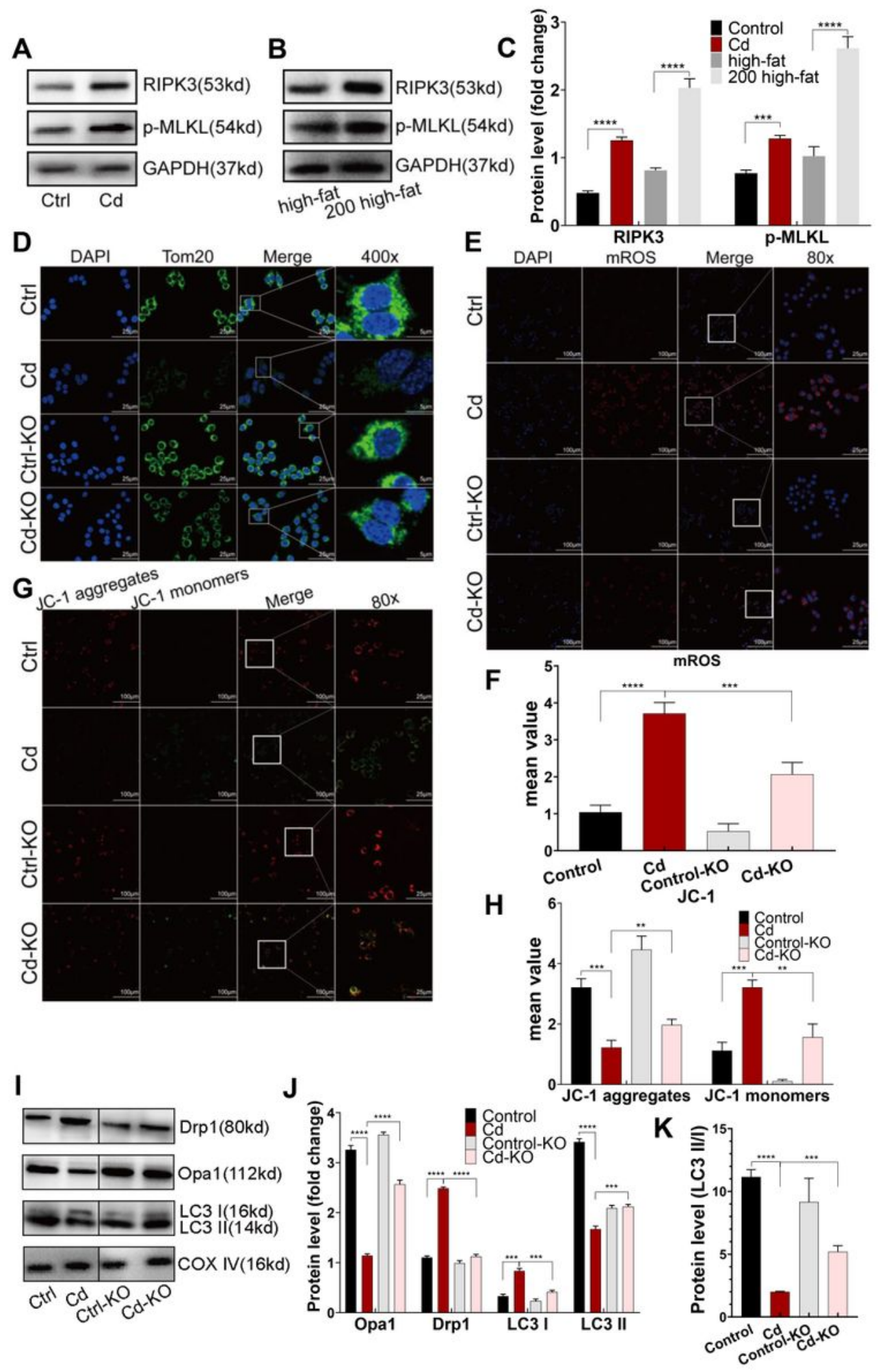

Figure 5 
The RIPK3-p-MLKL pathway regulated mitochondrial homeostasis in Cd-induced AS. (A) The protein expression levels of RIPK3 and p-MLKL in RAW264.7 cells. (B) The protein expression levels of RIPK3 in aortic arch of ApoE-/- mice. (C) The gray value of the proteins mentioned above. (D) Immunofluorescence images of mitochondrial membrance protein Tom20 in bone marrow-derived macrophages (BMDMs) of ApoE-/- or RIPK3-/-/ApoE-/-mice treated with Cd. (E, F) Immunofluorescence images and fluorescence intensity of mitochondrial superoxide (mROS) in BMDMs. $(G, H)$ Mitochondrial membrane potential stained with fluorescent probe (JC-1). (I, J) The protein expression levels of Opa1, Drp1, LC3I and LC3II in mitochondria of BMDMs treated with Cd. (K) The ratio of LC3II and LC3I indicating the autophagy level. Data are shown as mean \pm SD. ${ }^{\star} p<0.05,{ }^{\star \star} p<0.01,{ }^{\star \star \star} p<0.001,{ }^{\star \star \star \star} p<0.0001$. 

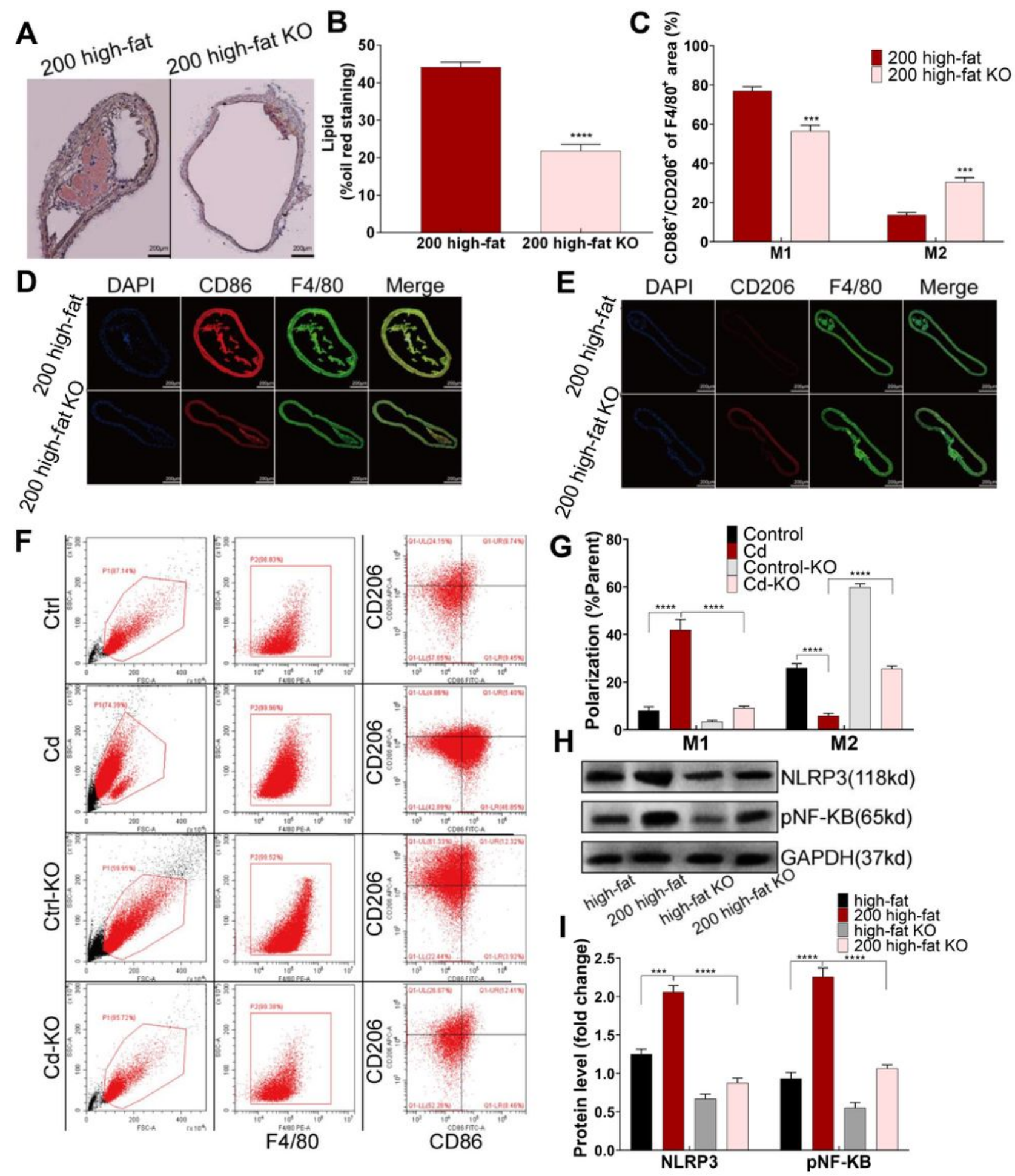

\section{Figure 6}

Deletion of RIPK3 inhibited polarity shift toward inflammatory macrophages and atherosclerosis. (A) Representative photomicrographs of the aortic arch segments from $\mathrm{Cd}$ - and high-fat-treated ApoE-/- or RIPK3-/-/ApoE-/- mice stained with Oil Red O. (B) Quantification of the atherosclerotic lipid content. (C-E) Aortic arch of Cd- and high-fat-treated ApoE-/- or RIPK3-/-/ApoE-/- mice were probed with specific antibodies against the macrophage marker F4/80 and co-probed with antibodies against the markers of 
M1 (CD86) or against the markers of M2 (CD206). $(F, G)$ The polarization of BMDMs treated by Cd showed by flow cytometry. $(\mathrm{H}, \mathrm{I})$ The protein expression levels of NLRP3 and pNF-KB in aortic root of mice after treatment with $\mathrm{Cd}$. $\left(\mathrm{n}=6-8\right.$ per group). Data are shown as mean $\pm \mathrm{SD}$. ${ }^{*} \mathrm{p}<0.05,{ }^{*} \mathrm{p}<0.01, * \star \star \mathrm{p}<$ $0.001, * \star \star \star x p<0.0001$.

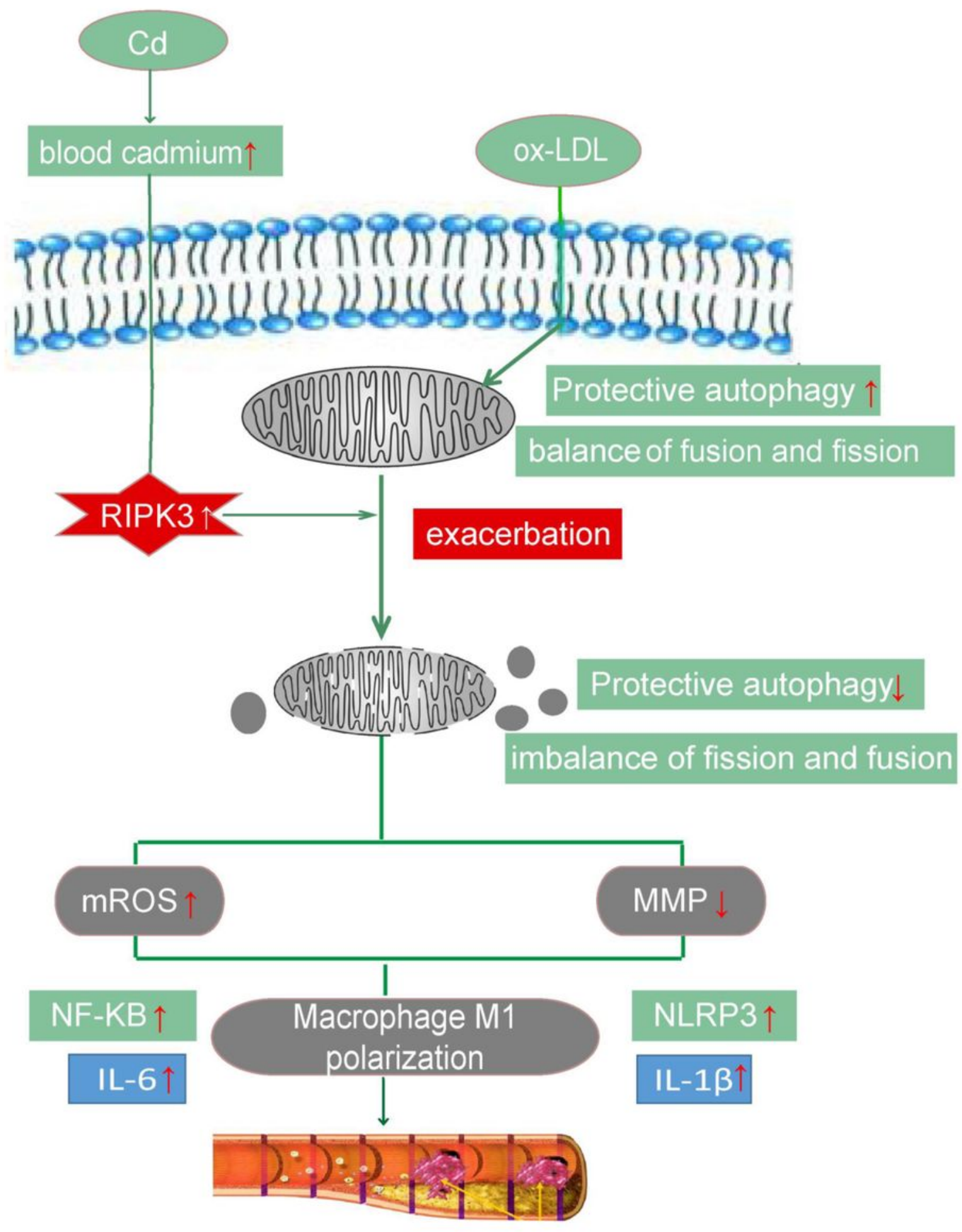

Figure 7

The mechanism of Cd-induced AS via RIPK3-regulated mitochondrial homeostasis 


\section{Supplementary Files}

This is a list of supplementary files associated with this preprint. Click to download.

- Additionalfile1.docx 